\section{$\S 14$. Boundary Modulation Effects on MHD Instabilities in Heliotrons}

Nakajima, N.

In three-dimensional configurations, the confinement region is surrounded by the stochastic magnetic field lines related to the separatrix or magnetic islands, leading to the fact that the various modulations of the plasma-vacuum boundary will be induced around the stochastic region by a large Shafranov shift of the whole plasma, in especially high- $\beta$ operations. To examine such modulation effects of the plasma boundary on MHD instabilities, high- $\beta$ plasmas allowing a large Shafranov shift are considered in the inward-shifted LHD configurations with the vacuum magnetic axis $R_{a x}$ of $3.6 \mathrm{~m}$, where pressure-driven modes are theoretically more unstable compared with experimental observations.

In the inward-shifted LHD configurations, the vacuum flux surfaces are so compressed into the helical coils inner side of torus that the bumpy deformation of the plasma boundary, expressed by the Fourier components with $(m, n)=(0, \neq 0)$ ( $m$ and $n$ are poloidal and toroidal mode numbers, respectively) is strongly enhanced. As $\beta$ increases, the whole plasma moves from inner side of the torus to the outer side. Through this Shafranov shift, the enforced boundary shaping by external coils will be so reduced that the bumpy deformation of the plasma boundary will diminish. In order to simulate the effects of the boundary modulation related to the bumpy components, currentless MHD equilibria with and without boundary modulation are investigated under the fixed boundary condition for various $\beta$-values. The boundary without modulation is determined from the outer most vacuum nested flux surfaces. The boundary modulation is created by eliminating only the bumpy Fourier components from the vacuum boundary spectrum. Broad pressure profiles, similar to experimentally obtained ones, are used. The ideal MHD stability analyses for compressible perturbations are performed by using the cas $3 \mathrm{~d} 3$ code.

Figure 1 shows the growth rates normalized by the Alfvén transit time on the magnetic axis $\gamma \tau_{A 0}$ vs the toroidal mode number $n$ for equilibria without boundary modulation (for typical high$\beta$ LHD operation parameters, $\gamma \tau_{A 0}=0.1$ corresponds to around $40 \mu \mathrm{sec}$ ). Figure 2 shows $\gamma \tau_{A 0}$ vs $n$ for equilibria with modulated boundary. Significant stabilizing effects by the boundary modulation are quite clear, which is due to a large Shafranov shift brought by eliminating bumpy components. Note that the experimentally observed modes with $(m, n)=(2,3)$ are weakly excited like external modes. The MHD equilibria with the boundary modulation of eliminating the bumpy components have many consistent properties to experimental observations.

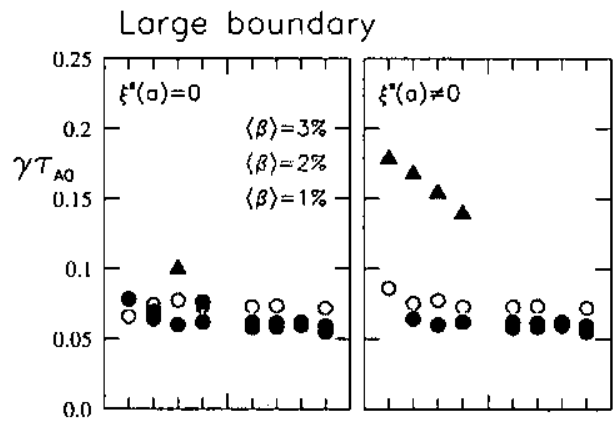

Figure 1: $\gamma \tau_{A 0}$ vs $n$ for equilibria with vacuum boundary. The left (right) column corresponds to fixed (free) boundary condition. The circles (triangles) denote interchange (ballooning) modes.

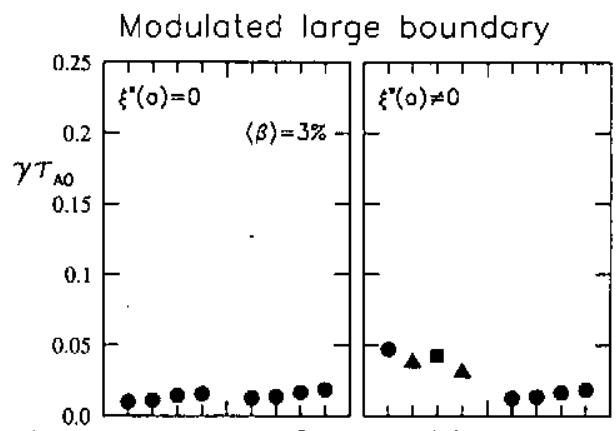

Figure 2: ${ }^{0} \gamma \tau_{A 0}$ vs $n$ for equilibrium with modulated boundary and $\langle\beta\rangle=3 \%$. Additional rectangles indicate ballooning modes with external components. 